\title{
On-demand Shared Digital Twins - An Information Architectural Model to Create Transparency in Collaborative Supply Networks
}

\author{
Jan Cirullies \\ FH Dortmund University of Applied Sciences and Arts \\ jan.cirullies@fh-dortmund.de \\ Fraunhofer ISST \\ jan.cirullies@isst.fraunhofer.de
}

\author{
Christian Schwede \\ FH Bielefeld University of Applied Sciences \\ christian.Schwede@fh-bielefeld.de \\ Fraunhofer ISST \\ christian.schwede@isst.fraunhofer.de
}

\begin{abstract}
Industrial companies already apply digital twins for the digital representation of the physical world. In addition, information sharing becomes increasingly decisive for the competition, e.g. in supply networks where products and logistics data such as demands and capacities are exchanged. As many companies are, however, highly reluctant to share data across the supply chain, this paper applies the methodology of design science research to, first, state the requirements for shared digital twins based on five industrial use cases. It turns out that with regard to data decentralism, sovereignty and compatibility through global standardization are key success factors. Hence, second, this paper presents a concept for a shared digital twin providing data on demand, i.e. at the right time and in particular with data condensed to the concrete need.
\end{abstract}

\section{Introduction}

Volatility, uncertainty, complexity and ambiguity (VUCA) are confronting our increasingly digitalized world and economy [4]. Hence, concepts for planning and controlling value creation systems in today's western economy have a high need of gain real-time flexibility to cope with the VUCA-induced challenges. Industry 4.0, which originates from Germany, and comparable initiatives from nearly all industrialized countries address this need with decentralized autonomously acting systems that are connected digitally in real-time in an internet of things [24]. The key factor of success of such a system is that the actors make their decisions based on transparency about an environment that exceeds their local horizon. The more relevant information is available to a smart device, the more foresighted and sustainable it can autonomously decide. Therefore, access to an end-toend supply chain digital twin is required and is the current approach in research and practice. One main task of supply chain management is demand and capacity management between the companies. Getting the right amount of parts for the current demands is a task that starts with long term matching of estimated demands with production and transportation capacities for parts and ends with short term fire-fighting of part procurement or adapting the production program in case of disruption to keep the production running. Finding a feasible solution in these highly interconnected networks, would need detailed data of each partner (e.g. capacities, costs of adaption measures, stocks). This data could be found in a digital twin of the network. However, creating a digital twin in decentral environments can only be achieved by constant data interchange of detailed information in near real-time between the autonomous actors leading to a shared digital twin. This is especially true in supply networks that have been decentral systems with autonomous actors since their existence.

Driven by the business need, data exchange in supply chain has been a central topic since their early days [7]. As demonstrated in this paper, a lot of initiatives of the past have tried to establish a digital twin for supply networks and mostly failed. Hence, this paper aims to answer two research questions:

1. Why did past initiatives succeed or fail and, in conclusion, what are the requirements for a shared digital twin in collaborative supply networks?

2. How must a conceptual approach be designed to enable a highly collaborative on-demand shared digital twin?

In this paper, reasons for these failures are presented by analyzing multiple industrial cases (4). Based on these findings, requirements for a shared digital twin for collaborative supply networks are derived (5) followed by the presentation of an information architecture for an on-demand shared digital twin architecture in decentral environments that cope with these requirements (6). Finally, section 7 draws a conclusion and outlines further research need. 


\section{Research methodology}

The methodology for the information architecture development starting with the use cases selection is presented in this section. The use cases to be analyzed, first, must be related to the development of a shared digital twin for supply networks. Second, sufficiently deep insights were required to be able to draw differentiated conclusions on success factors and expected barriers. Hence, more than 50 of the industry-related research projects in the field of supply chain digitalization that the authors have conducted were assessed with the following criteria: (1) multilateral both intra- and cross-company data exchange, (2) data integration/compatibility and (3) data security/sovereignty. In consequence, five use cases are considered in this paper, each one with individual focus areas like data processing and visualization, particular purposes (e.g. risk management) or data sovereignty. It turned out that all cases are from the automotive industry. This is plausible as this branch is often considered as an innovation driver due to its high product and process complexity. Although all of the finished projects had respectable outcomes, they did not achieve a commonly accepted and in industry adapted model or architecture to enable a shared digital twin.

The methodological approach of a case study is suitable to answer the two research questions based on the selected use cases as a case study answers question types of "how" and "why" that do not require control over behavioral events (e.g. influencing decisions made in the use cases) and focus on recent events [42]. Hence, this method is applied to identify requirements (research question 1) and to derive a conceptual solution approach (question 2). According to Gustafsson [17], there are two types of case studies: single- and multiple-case studies. As soon as more than one case is analyzed, a multiple-case study has to be carried out [37]. The choice of multiple use cases allows the replication of results across use cases which is one advantage over single-case studies [42].

The objective of the research in this paper to develop an information architecture model for ondemand shared digital twins. Methods and models are a typical artifact in design science research. Three levels of contribution and abstraction characterize an artifact [14]. Level 2 comprises general (abstract) contributions such as models and methods and is, thus, intended in this paper.

The framework according to Hevner et al. [21] and Hevner [20] defines three cycles for artifact development: relevance, design, and rigorousness. In this paper, the relevance cycle is covered partially by the presentation of the industrial cases. The theoretical foundation in this paper covers the full cycle of rigorousness because foundational models and methods are considered to form the artifact which, in turn, then enhances the knowledge base again. The design cycle is performed by the information architecture development (concept section) and the justification in the conclusions. Starting with the knowledge application as part of the rigorousness cycle, section 3 presents the theoretical foundation.

\section{Theoretical foundation}

Mentzer et al. define supply chains "as a set of three or more entities (organizations or individuals) directly involved in the upstream and downstream flows of products, services, finances, and/or information from a source to a customer" [29]. Supply networks emphasize the immanent complexity, as supply is not provided in a linear chain, but in branched relations. The according management discipline, supply chain management (SCM), is the concept of coordinating information and material between companies [7].

As the remark on information exchange already indicates, also Hofman and Dalmolen state that "data sharing and interoperability are a prerequisite for decision support by individual actors that are hyperconnected" [22]. As supply chains can be distinguished between intra- (process management within the same organization) and inter-organizational scope (cross-supply-chain) [8], the review of relevant technologies is structured accordingly.

\subsection{Cross-organizational technologies}

In the last decade, the term "digital twin" gained increasing attention in industry and enterprise management. Definitions and perception of digital twins varies, but in a very recent as well as sophisticated literature survey, van der Valk et al. [39] conclude, that a definition of a digital twin given by Tao et al. covers most characteristics of digital twins discussed in literature [38]: A digital Twin consists of a physical element, virtual element (in the case by Tao et al.: products) and connected data that tie the two elements. The digital twin comprises "(1) real-time reflection [...], (2) interaction and convergence [...], (3) self-evolution". But the same review also comes to the conclusion that there are only two papers addressing digital twins for logistics so far. One was written by one of the authors and was not focused on the topic of data retrieval [27], the other is focusing on product digitization during transport [10].

Recent research stresses the aspect of multilateralism, thus, speaking of a "shared digital 
twin" and deriving further need for research [6]: What are appropriate query approaches to retrieve shared digital twin data and what are appropriate synchronization approaches for distributed data? "Distributed data" can, on the one hand, relate to storage clusters and parallel computing which usually comprises a central orchestrator for assigning data and jobs to the nodes (cf. e.g. [28]), gaining further attention because of the currently high attention on hyper ledgers. However, on the other hand, distributed or "decentral" data can also refer to data that is independently managed and stored, but may be relevant as a whole for certain issues [34].

The semantic web solves the challenges resulting from the need for linked data. Technologies like the Resource Description Framework (RDF) and the Web Ontology Language (OWL) enrich data by semantic to make them machine readable. An ontology "includes a vocabulary together with a specification of the intended interpretations (meanings) of the terms in the vocabulary" [16].

In the area of supply chains, Grubic and Fan [15] conducted a systematic literature review and identified (in 2010) six ontology models. More recently, due to data heterogeneity in supply chains, e.g. Nimmagadda et al. [31] presented a model for big-data-guided supply chains in knowledge-based geographic information systems including "multidimensional ontologies [...] facilitating the relationships between events of supply chain operations". Also Hofman and Dalmolen [22] base their research on "data sharing in supply and logistics networks" on ontologies to ensure "extendable, standardized platform services for [...] in an open dynamic ecosystem of organizations".

A quantitative and exemplary literature survey by the authors showed that in the last five years the growth rate of the number of publications matching the expressions "supply chain" and "ontology" increased from $5 \%$ to $11 \%$ per anno. Evidently, ontologies and linked data play an increasing role in supply chains, at least from a research perspective.

Heath and Bizer distinguish three general architectural patterns for linked data in an overview [19]: (1) The crawling pattern is "suitable for implementing applications on top of an open, growing set of sources" by storing a local cache providing reasonable performance for complex queries on big data." (2) The on-the-fly dereferencing pattern is based on queries dereferencing links at runtime, thus, avoiding copies of data and therefore possibly outdated data (e.g. in a cache). However, performance can be very low. (3) In the query federation pattern, complex queries are sent to the data source and executed. The performance may also be very low.
One important recent European initiative to data interchange between companies are the International Data Spaces (IDS) connecting data owners and users via on-demand peer-to-peer connections (Figure 1).

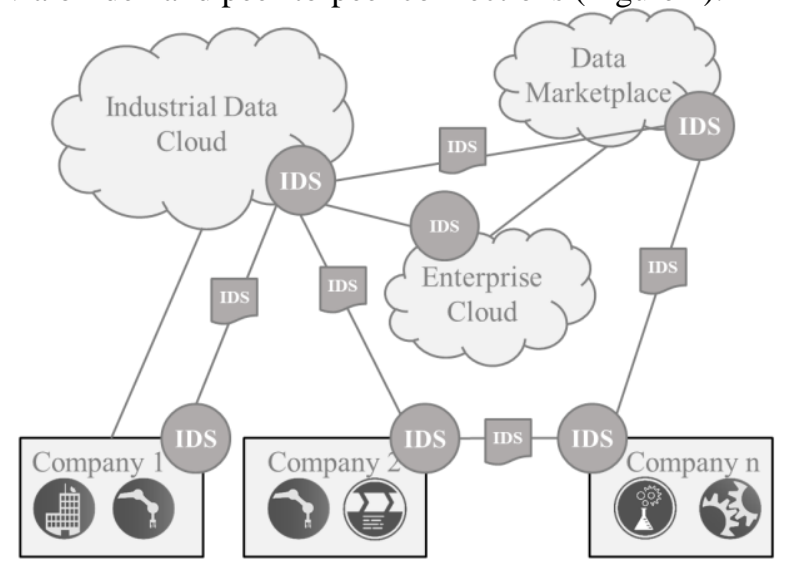

Legend: (-) IDS Connector; -DSt-Usage Constraints; — Non-IDS Communication.

Figure 1. International Data Space architecture for sovereign data exchange

When data is provided either by the data owner/creator itself or by an intermediary (service provider, possibly a platform), data is exchanged through a software gateway called IDS connector. The connector provides initially a self-description according to the IDS information model containing meta data, accessibility information, eventually prices, usage policies etc. Usage policies are based on the Open Digital Rights Language (ODRL). If data is requested and the request is authorized, the connector provides the data content to the requesting connector. The architecture of the IDS connector [33] consists among others of an application container management, a communication bus and a configuration manager. A container-based application (or a chain of many of them) in the connector can, first, process received data [30]. As a certified connector is to be considered to be trustworthy, it will enforce usage policies and provide incoming data only to the authorized applications.

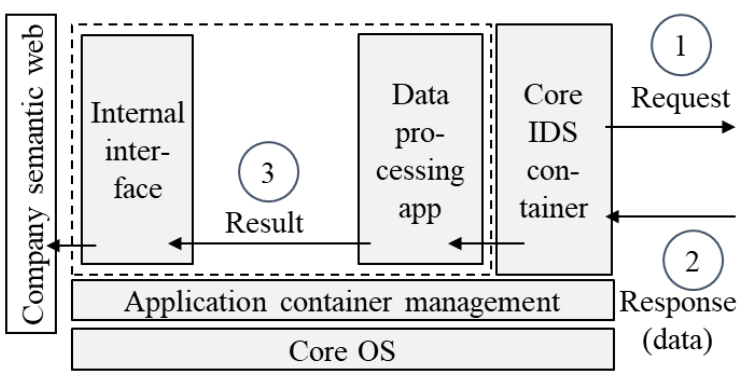

Figure 2. IDS connector (schematic) of a supply network participant 
Second, if data is provided, these apps can perform data pre-processing (aggregate, anonymize or in any other way calculate data). Figure 2 outlines the operation of the IDS connector for data receiving.

Processing data in an agreed (and potentially trusted) IDS connector app (e.g. "data processing app" in this figure) can be declared mandatory in the terms of use in the IDS vocabulary. In consequence, only the processed data and therefore the potentially sufficiently less critical result is forwarded to the next IT services in the processing chain.

The applicability of IDS for industrial purposes was demonstrated e.g. by Zrenner et al. [43]. iShare [9] is a comparable architecture with similar goals.

\subsection{Intra-organizational technologies}

Companies see cross-company data exchange critically. First, data sharing bears a certain risk (e.g. of data abuse) that is differently perceived and mitigated by the individual manager [23]. Second, not only creating data at all, but also providing it at the right quality [11], creates higher effort (although it brings also internal benefits). According superimposing levels of digital maturity provides e.g. the acatech Industry 4.0 Maturity Index [35] distinguishing between visibility, transparency, predictive capability and adaptability. This means, companies have to gain these digital capabilities stepwise to act autonomously and self-optimizing.

Technically, adequate data management and processing is required. If, as in many cases, data is gathered from the intra-organizational processes, it is directly used in the same organization. This, first, allows pragmatic implementation without the need to stick to global data standards. Second, investing in data generation is usually simple to assess and decide as effort and benefit affect the same organizational instance. Shared (cross-organizational) digital twins, however, mostly lead to organization-wise separated effort and benefit [2]. Therefore, organizations additionally need motivation for data sharing (measurable benefits for operations, monetary compensation, strategic benefits etc.).

According to the methodology, the findings from the literature survey are mirrored with the authors' findings and experiences from industrial application (relevance cycle).

\section{Use case for data sharing in supply networks}

Based on long-term experience of the authors, five use cases are presented that aimed for a digital twin in supply networks. The use cases are then analyzed regarding success and barriers in the following.

In the publicly funded project ViLoMa (visual logistics management; finished in 2016), one automotive original equipment manufacturer (OEM), two 1st-tier suppliers and three logistics service providers participated to provide complete data sets with intuitively usable web interfaces to logistics planners and operators [3]. IT architecture was based on a central data repository (SAP HANA). The consortium highly agreed on the necessity and purpose of the project, and remarkable progress was achieved toward ergonomics and (proprietary) integrated meta data models. However, data integration remained on an exemplary level. The most critical barrier was that the partners were reluctant to share data in a central data pool which is interesting and relevant for the partners in the intended scenarios, but also potentially critical for the data provider. Especially providing detailed data such as available capacities continuously without a concrete incidence such as a bottleneck or disturbance was seen as a problem. In consequence, missing trust was an important limiting factor. In addition, long clearing processes indicated that some project participants were not prepared to manage data sharing from an organizational/technological perspective. Thus, the immediate scaling potential from this small supply chain fraction to multi-tier supply network was rather low due to organizational matters and data integration.

In the project RAN (RFID-based automotive network; finished in 2012) two automotive OEM and several material and IT suppliers jointly enhanced the existing EPC (electronic product code) based on the requirements of the automotive industry and developed the info broker, an enhancement of the EPC global network that allows to share data in a standardized way according to adjustable data access rules [41]. The project built upon existing standards (such as EPC) to increase scalability and acceptance which then, positively, lead to the integration of the findings into the standard VDA 5500 of the German automotive industry association. However, the concept of data sharing through the info broker was not established. Therefore, the successful data format standardization refers to comparably uncritical data like location of a container or a finished vehicle. These data may form the basis of a shared digital twin, but do not yet fulfill a business purpose without further context data (e.g. to detect delays, issue smart payments etc.). A difficulty was the reluctance of the partners to adapt their internal data formats and IT standards. Consequently, the agreement on a standard took about eight years. 
A more advanced level of collaboration with focus on risk management was developed in the project InkoRisk (integrated scheduling and planning in complex network structures; finished in 2013). One essential outcome were two inter-organizational supply chain risk management process [13]. One process aimed to exchange needed data and possible countermeasures in acute situations (active measures process). The second process defined regular consultation of relevant parties to review occurring risks, define standard counter-measures and decide on essential (structural) improvements (passive measures process). IT-wise, the idea was again to store data in a central risk data repository which lead to the same problems of partners not willing to provide critical data continuously in a central data base.

Based on the data security mechanisms and standards of the International Data Space (IDS), Volkswagen is setting up decentral data exchange for demand and capacity data in the supply chain (ongoing) [5]. The project ensures data sovereignty for data providers, because, first, data is stored in the sphere of the data provider and, second, terms of use can be attached to each data object that are enforced by the receiving data gateway. As these mechanism form a trustworthy environment for sharing, the partners share internal capacity data that are, on the one hand, highly critical from the provider's view, but, on the other hand, very useful for the receiver to lever planning flexibility potential. The vision hence comprises the idea of a recursive demand capacity check in the entire supply network [32].

Current projects from large German and Swedish automotive OEM demonstrate the relevance of shared digital twins also within organizations. The most prominent example for big data analytics is predictive maintenance. In large companies with many sites, data management becomes an enabler for business process improvements, because each entity describes data in an individual way and is reluctant to share data even within the organization due to insufficiently defined clearance processes. Again, missing standardization in meta data and sharing processes interfere with the goal of a group-wide shared digital twin.

\section{Problem analysis and requirements}

Firstly, analyzing the different case studies shows that a central misunderstanding of creating one overall digital twin for a supply network is the assumption that this would even be possible. Since the idea of a digital twin originated in locally and centrally organized areas such as product development and production planning, the perception that one could build it in the same central manner for a supply network seems logical.
First of all there is not one supply network, but in fact hundreds of them, one for every participant with their respective suppliers and customers. Creating one central digital twin of the supply chain would rather mean either focusing on only one participant or trying to create a digital twin for the whole industry. Putting one supply chain partner in focus appears obvious for a focal firm as such a company guarantees a high impact. However, usually this company also benefits most, thus keeping the motivation low for other partners to share data. The second option to create one digital twin for a whole sector in a single repository would be technologically possible, but would also pose the issue of immense power agglomeration at one instance. Cases like ViLoMa and InKoRisk both failed using this central approach with the local view of a specific partner (in these cases the OEM).

Secondly, the aim of permanently interchanging data to create a constantly up-to-date digital twin is especially difficult when data is critical and can be used against one partner (e.g. capacity information, costs). Case study InKoRisk shows this exemplarily: Even though detailed information in the context of risk management is mainly relevant in the case of an incident, the aim was to permanently interchange these information leading to unsolvable caveats.

Thirdly, partners seem to be willing to share critical data if they know that it will not be used against them as shown in the IDS case study. Fourthly, the necessity to adapt local data formats to a global standard seem to be a major problem for companies to share data over their company boundaries. Case study RAN showed that the main preventer were the local data standards. Even though only completely none critical data of transport events were addressed. Fifthly, case study International Data Spaces and RAN show that it is possible to spread a solution throughout the network if common standards are defined.

Summing up the following requirements for digital twins in supply networks are derived:

RQ1: Digital twins have to be decentral since the supply network is decentral

RQ2: Digital twins have to be created on demand and adapted to the current situation to minimize data interchange requirements

RQ3: Sovereignty about how the data is used has to be guaranteed.

RQ4: Shared data for the twin should be independent from local data formats

RQ5: Data interchange should nevertheless follow a global standard for easy usage in the whole network

From these requirements, a concept for an ondemand shared digital twin for supply networks is derived in the following section. 


\section{Concept of an on-demand shared digital twin}

The case analysis and the concluded requirements outlined the special need beyond a digital twin for limited areas such as product development or production planning. As motivated in section 3, supply chain data interchange has to consider two perspectives. First, the concept from a supply network perspective is developed (6.1) and requirements for the intra-organizational data management are derived. After that the concept for the intra-organizational perspective is described accordingly (6.2). This represents the design cycle.

\subsection{Supply network perspective}

From the five requirements result two major design concepts on the supply network perspective. To interchange data, we need a common language (architecture component A) for all the participants as well as an infrastructure (architecture component B) to connect them (cf. sec. 3). Both language and infrastructure have to address the complete set of requirements stated in section 5 .

Ontologies form the state of the art for the language component (A) and are especially based on RDF (cf. sec. 3). They allow a common (crossorganizational standardized) data description and are, thus, suitable to provide a common description of supply chain data for particular purposes. Therefore, they address two of the five requirements in particular:

First, as the purpose of ontologies is to make data "understandable" and linkable. The competition among ontologies controls the number of relevant ontologies for one domain, thus, defining a quasistandard for data description [26]. Publicly available ontologies can be retrieved e.g. from DBpedia (cf. 3.1). Hence, ontologies basically fulfill RQ5.

Second, ontologies can be easily scaled and extended to a focused subdomain without invaliding the data model thus addressing RQ2, i.e. a digital twin adapted to the immediate need. As ontologies allow all parties to directly "understand" data, they enable building an on-demand digital twin [36]. Choosing the right part of the ontology ensures the adaption of the twin to the immediate situation [40]. E.g. a particular digital twin for bottleneck management would not have to comprise capacities for a whole time series, but only - in the minimum case - an information "bottleneck can be solved / not solved".

To address the remaining three requirements concerning the connection to local data, the language has to enable access to that data. Therefore the ontology is combined with meta data that describes where to find the data (RQ1 and RQ4) and what is allowed to do with the data (RQ3). Since the supply network perspective can only address the interchange between the boundaries of the companies, there are additional requirements for the intra-organizational perspective (cf. sec. 6.2) needed:

RQ1_A1: Connection of RDF-based requests to the local data source to locate data within the company and integrate received data

RQ2_A1: Definition of usage rules to consider data sovereignty

RQ3_A1: Mapping of RDF to local data sources to ensure independency from local data formats

There are only few technologies or architectures like iShare, IDS or ODRL that fulfill the requirements presented above to a certain extent. One and as far as known the only suitable combination of architectures is the IDS in combination with ODRL. In comparison to iShare, IDS creates a whole ecosystem with roles and software components, especially gateways that allow remote usage policy enforcement. Furthermore, there is a large and international community behind it.

Data in the IDS is described based on the RDFbased IDS ontology [33]. This ontology (1) combines meta data with local domain-specific ontologies and (2) allows to describe data and its sources for seamless adaptability. It (3) includes usage policies based on ODRL that can be attached as a part of the meta data defining e.g. by whom, till when or for which purpose data may be used or not used. Appropriately, the IDS vocabulary only comprises all aspects of data retrieval, exchange, policies etc., thus, "domain modeling is delegated to shared vocabularies and data schemata" [33]. Thus, it explicitly enables ontology-based domain-specific vocabularies.

By that, the IDS perfectly complements the language concept described above and contributes to fulfill the requirements RQ 2, RQ 3 and RQ5. Furthermore, decentral data is localized and interchanged by the IDS connectors (RQ1) which also allows to be connected to the local data sources (RQ4). However, this has to be achieved by the respective supply chain partner. Therefore, the additional requirement for the company perspective applies:

RQ1_B1: Implementation of IDS Connector and connection to local sources

An IDS connector is retrieved by a unique address that can either be looked up in the data broker or (more common in industrial cases) is shared between the supply chain partners initially manually. This also implies one more company-related requirement:

RQ1_B2: Offer data services in the data broker or directly with partners 
At this point, the connection between language and infrastructure is still missing: As presented in the theoretical foundations, three linked data access patterns can be distinguished in general [19]. Indeed, the crawling pattern promises highest performance. However, considering RQ2 and RQ3 (ad hoc linking and data sovereignty), the immanent data cache endangers the enforcement of data terms of use. Also outdated data might occur. The effort for complex supply chains (as automotive) is considered as comparably low: First, there might be thousands of (sub-)suppliers for one OEM providing thousands of parts. However the number of critical stock-keeping is rather low. Second, the complexity of queries is expected to be rather low as, e.g. in the case of bottleneck management, simple additions of few integer values are necessary. Therefore, the two other patterns for linked data, appear applicable.

Considering the required data sovereignty (RQ3), both patterns can contribute their value: The on-the-fly dereferencing pattern ensures direct data processing without unwanted copies in the local cache. The query federation pattern can be applied for highly confidential data that shall be processed at the data source owner. Appropriately, these two patterns match the architecture and operation schemes of the IDS.

In summary, ontologies for the domain of supply chain linked by the RDF-based IDS ontology scheme form the language component (A) of the information architecture and the IDS architecture services as the basis for the infrastructure (B). In combination, they are highly suitable to enable on-demand shared digital twins fulfilling all five requirements for the supply network perspective. However, this leads to additional prerequisites for the company perspective.

Figure 3 shows the approach from an on-demand shared digital twin based on the IDS: Each participant of the supply network has one (or more) IDS connector with a unique address. The data is shared based on the open supply network ontology stored in the ontology repository and connected to meta data defined by the IDS architecture. IDS connectors (and specific data services) can be found using the data broker or already shared addresses. Based on this approach the relevant part of a shared digital twin is created on-demand and by sharing only the relevant and needed data.

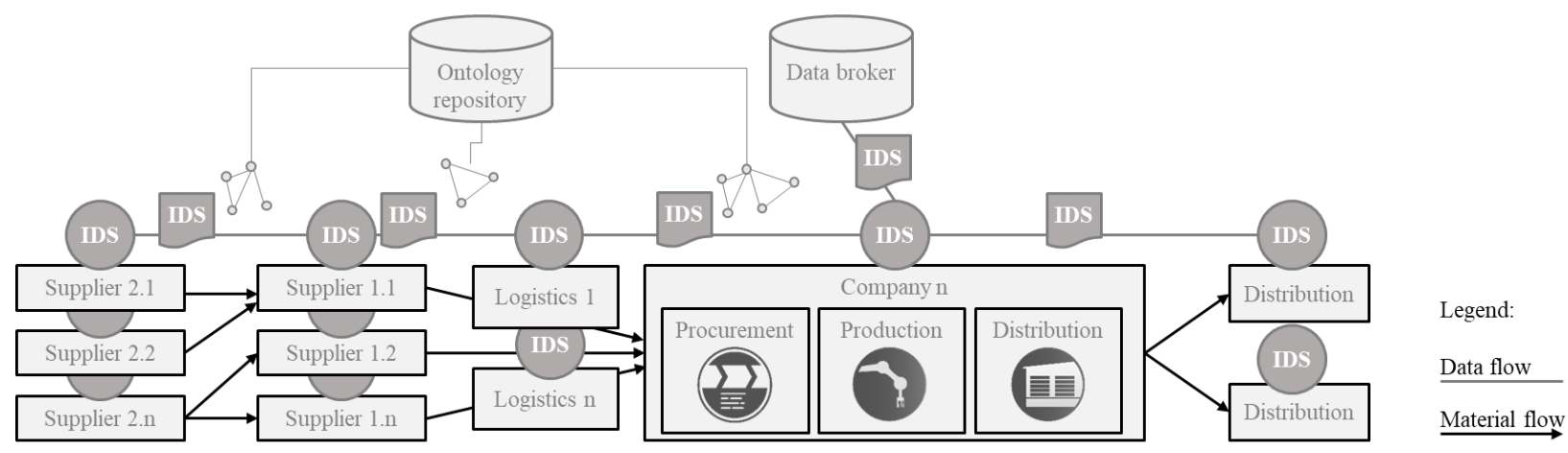

Figure 3. Approach for an on-demand shared digital twin from the supply network perspective

Besides this network perspective, also the individual participants (i.e. supply network partners) have to contribute to fulfill the requirements. The according concept is described in the next sub-section.

\subsection{Company perspective}

The previous part of the concept described the mechanisms for data exchange on the network level. To take part in this requirements-fulfilling concept, companies have to enable themselves technically and organizationally to both contribute to and benefit from a shared digital twin.

The creation of data, ranging from sensing on the shop floor via IT system input by humans to bilateral, (electronic) data interchange, is considered as a prerequisite for a digital twin and, thus, not outlined in this concept. Shop floor data ("IoT data") usually passes several processing layers from the edge device through a middleware to an IT system (see e.g. [18]). Transactional data is, especially in larger mediumsized and large companies, usually available in various management systems such as enterprise resource planning systems (ERP), warehouse management systems (WMS), etc. In most cases, however, data compatibility even between departments or business units is low. Since the task in the context of supply network data interchange is to provide the data to the partner (RQ1_A1, RQ3_A1), one major challenge is the internal data organization. Companies are challenged to find a solution between, on the one hand, pragmatically developed, but incompatible individual installations and, on the other hand, highly compatible, all-standards-compliant information systems and data 
models that may not fit local processes and needs perfectly, therefore, causing high effort for changes.

For the supply chain perspective, this paper proposes that supply chain participants map relevant information resources internally to suitable and broadly accepted ontologies. The applicability for industrial application in the context of Industry 4.0 was proven e.g. by Bader et al. [1]. The ontology should be connected to meta data and, thus, separating the "physical" data storage from the data access layer. First, this combines both individual development on the data source and local IT system level as well as standardized and compatible data models on the access layer. Second, the often requested internal central "data lake" is avoided by this approach reducing redundancy in data storage or data dumps [25].

The meta data model in the proposed approach would comprise: (1) functional semantic description of the data, (2) usage rights for intra-organization but especially for supply-chain-wide use (RQ2_A1) and (3) information about the accessibility (URI, authorization conditions and procedure). As the links between these data and their semantic meaning should be encoded, storing them as RDF triples in a triplestore is proposed. Meta data may be stored in a data catalog which is a data base that describes available data objects, their location and access procedure as well as the usage rights.

The resulting architecture is depicted in Figure 4 based on the foundation of Heath and Bizer [19]. Available data is prepared for linked data by a wrapper which contributes to the company-internal semantic web (RQ3_A1). Relevant meta data is included from the data catalog including usage rules (RQ2_A1). IT services are allocated on the data access layer providing data to both internal (domain-specific) applications and the shared data model in the company. The IDS connector (RQ1_B1), as part of the proposed shared digital twin architecture, is assigned to the data access layer, too, as it represents a piece of infrastructure fulfilling the required feature of data sovereignty by respecting the terms of use from the data catalog annotated to the data. The connectors also apply internal and external ontologies enabling reaction on data requests (RQ1_A1) and integrating provided data into the internal data format (RQ1_A1).

This internal architectural perspective complements the classification of the IDS in a typical enterprise architecture stack [33]: the architecture below includes the "vertical" data integration from processing of internet things via basic services to the domain-specific application whereas the IDS is allocated on the services level (data access level) connecting domains and organizations.
The proposed company-internal architecture in Figure 4 provides data to external application through the IDS connector (solid directed edges). The required data is read from the meta data model consisting of data from the respective IT systems and meta data from the data catalog. Data receiving and integration (dashed lines) works analogously: The IDS connector enriches the meta data model with data from external sources. For the company remains the task to offer their data services publicly using the data broker or sending the corresponding service addresses to their partners (RQ1_B2).

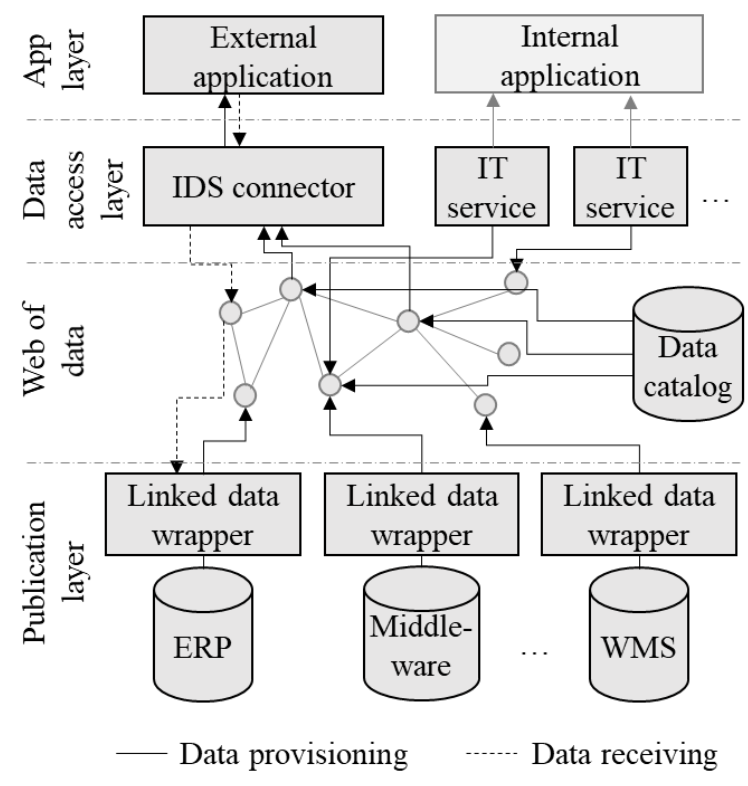

Figure 4. Shared digital twin architecture from the company perspective

\section{Conclusions}

Since the topic of digital twins in rather new in the area of supply networks and there are only very few papers available on this topic, this paper derived an architectural model for on-demand transparency in collaborative supply networks based on the challenges of projects on shared digital twins. The model was created to fulfill five requirements (RQ1-RQ5) that were derived from five industrial use-cases. These requirements have to be reflected and tested against further use cases by the scientific community. As a result, the model allows decentral, on-demand data exchange where participants keep the sovereignty over their data, exchange data only when needed and keep their independence from central infrastructures. Furthermore, it follows global data standards without the necessity to change local internal data formats. To 
cover all requirements, the model was split into a supply network and a company perspective and based on the reference architecture of IDS. While IDS has been proven to be accepted in practice, ontologies are not yet well-accepted in industrial application [12]. Guidelines may help to overcome this problem, but, according to the experience of the authors, enforcing implementations and application in broader contexts is the most effective and finally necessary way. Another issue is the data quality within companies. Due to the ongoing digital revolution, the importance of data quality increases requiring proper data management processes within companies including data governance systems. At last the known problem of cost benefit sharing in supply networks can be still blocking implementations as the supply chain partner creating data often does not benefit from it.

Thus, there is still need for research. Especially the topic of data value has to be addressed more specifically. Still the question of how to micro-manage prices or more in general the question of how to micromanage what to share under with conditions with whom must be answered. The authors believe that in decentral autonomous systems software agents will be key actors to manage information trade in supply networks. Finally, to proof the functionality of the proposed architectural model, it has to be implemented and tested in concrete use cases. The IDS ecosystem would be a preferred start to identify partners from industry, technology and research.

\section{Acknowledgements}

This research was supported by the European Commission in the Horizon 2020 project Trusted Secure Data Sharing Space (TRUSTS), grant agreement no. 871481 .

\section{References}

[1] Bader, S., I. Gragel-González, P. Nanjappa, M.-E. Vidal, and M. Maleshkova, "A Knowledge Graph for Industry 4.0", in The Semantic Web, A. Harth, S. Kirrane, A.-C. Ngonga Ngomo, H. Paulheim, A. Rula, and A.L. Gentile, Editors, Heraklion, Crete, Greece, May 31-June 4, 2020. 2020. Springer International Publishing: Cham.

[2] Barua, A., S. Ravindran, and A.B. Whinston, "Enabling information sharing within organizations", Information Technology and Management, 8(1), 2007, pp. 31-45.

[3] Beißert, U., M. Reinbold, C. Beyer, and A. Sydow, VILOMA - Visual Logistics Management project, 2016.

[4] Bennett, N. and G. Lemoine, "What a difference a word makes: Understanding threats to performance in a VUCA world", Business Horizons, 57(3), 2014, pp. 311-317.
[5] Burkhardt, U., K. Borrmann, and S. Opriel, IDS@BKM: Gaining Transparency in Automotive Supply Chains, Paris, France, 4.12.2019.

[6] Capiello, C., A. Gal, M. Jarke, and J. Rehof, "Data Ecosystems: Sovereign Data Exchange among Organizations (Dagstuhl Seminar 19391)", Dagstuhl Reports, 9(9), 2020, pp. 66-134.

[7] Chopra, S. and P. Meindl, Supply Chain Management: Strategy, Planning, and Operation, 6th edn., Pearson, Boston, 2016.

[8] Cousins, P.D. and R. Spekman, "Strategic supply and the management of inter- and intra-organisational relationships", Journal of Purchasing and Supply Management, 9(1), 2003, pp. 19-29.

[9] Dalmolen, S., H. Bastiaansen, M. Kollenstart, and M. Punter, "Infrastructural Sovereignty over Agreement and Transaction Data ('Metadata') in an Open Network-Model for Multilateral Sharing of Sensitive Data", in Fortieth International Conference on Information Systems, ICIS, Munich, Germany. 2019.

[10] Defraeye, T., G. Tagliavini, W. Wu, K. Prawiranto, S. Schudel, M. Assefa Kerisima, P. Verboven, and A. Bühlmann, "Digital twins probe into food cooling and biochemical quality changes for reducing losses in refrigerated supply chains", Resources, Conservation and Recycling, 149, 2019, pp. 778-794.

[11] Falge, C., B. Otto, and H. Österle, "Data Quality Requirements of Collaborative Business Processes", in 2012 45th Hawaii International Conference on System Sciences, 2012 45th Hawaii International Conference on System Sciences (HICSS), Maui, HI, USA, 04.01.2012 07.01.2012. IEEE.

[12] Feilmayr, C. and W. Wöß, "An analysis of ontologies and their success factors for application to business", Data \& Knowledge Engineering, 101, 2016, pp. 1-23.

[13] Gelau, T., J. Cirullies, C. Schwede, A. Kirazli, and R. Schwindt, "A conceptual approach to collaborative supply chain risk management in the automobile industry", in Proceedings of the 21st EurOMA Conference: Operations Management in an Innovation Economy, 21st

EurOMA Conference, Palermo, Italy, 20.-25.6.2014. 2014.

[14] Gregor, S. and A.R. Hevner, "Positioning and Presenting Design Science Research for Maximum Impact", MIS Quarterly, 37(2), 2013, pp. 337-355.

[15] Grubic, T. and I.-S. Fan, "Supply chain ontology: Review, analysis and synthesis", Computers in Industry, 61(8), 2010, pp. 776-786.

[16] Gruninger, M., O. Bodenreider, F. Olken, L. Obrst, and P. Yim, "Ontology Summit 2007 - Ontology, taxonomy, folksonomy: Understanding the distinctions", Applied Ontology, 3(3), 2008, pp. 191-200.

[17] Gustafsson, J., Single case studies vs. multiple case studies: A comparative study. 
[18] Haße, H., B. Li, N. Weißenberg, J. Cirullies, and B. Otto, "Digital twin for real-time data processing in logistics", in Proceedings of the Hamburg International Conference of Logistics (HICL): Innovative Approaches for Supply Chains, W. Kersten, T. Blecker, and C.M. Ringle, Editors, Hamburg International Conference of Logistics (HICL), Hamburg. 2019. epubli: Berlin.

[19] Heath, T. and C. Bizer, Linked Data: Evolving the Web into a Global Data Space, 1st edn., Morgan \& Claypool, 2011.

[20] Hevner, A.R., "A Three Cycle View of Design Science Research", Scandinavian Journal of Information Systems, 19(2), 2007.

[21] Hevner, A.R., S.T. March, and J. Park, "Design Science in Information Systems Research", MIS Quarterly, 28(1), 2004, pp. 75-105.

[22] Hofman, W. and S. Dalmolen, "Data sharing in supply and logistics networks", in Proceedings of the IPIC 2019, 6th International Physical Internet Conference 2019 (IPIC2019), London, July 9-11, 2019. 2019.

[23] Huong Tran, T.T., P. Childerhouse, and E. Deakins, "Supply chain information sharing: challenges and risk mitigation strategies", Journal of Manufacturing Technology Management, 27(8), 2016, pp. 1102-1126.

[24] Industrie 4.0 in a Global Context: Strategies for Cooperating with International Partners, acatech - National Academy of Science and Engineering, 2016.

[25] Kachaoui, J. and A. Belangour, "Challenges and Benefits of Deploying Big Data Storage Solution", in Proceedings of the New Challenges in Data Sciences, R. Touahni, R. Messoussi, T. Boujiha, and K. Lekdioui, Editors, Acts of the Second Conference of the Moroccan Classification Society on ZZZ - SMC '19, Kenitra, Morocco, 28.03.-29.03.2019. 2019. ACM Press: New York, NY, USA.

[26] Kariapper, A.R. and P.M. Jayaweera, "Ontology is a Solution in Different Domains and Comparison of Successful Ontologies", in 2015 International Conference on Advances in ICT for Emerging Regions (ICTer).

[27] Korth, B., C. Schwede, and M. Zajac, "Simulationready digital twin for realtime management of logistics systems", in 2018 IEEE International Conference on Big Data, Seattle, WA, USA, 10.12.2018 - 13.12.2018.

[28] Li, S., M. Maddah-Ali, Q. Yu, and A.S. Avestimehr, "A Fundamental Tradeoff Between Computation and Communication in Distributed Computing", IEEE Transactions on Inform. Theory, 64(1), 2018, pp. 109-128.

[29] Mentzer, J.T., W. DeWitt, J.S. Keebler, S. Min, and N.W. Nix, "Defining Supply Chain Management", Journal of Business Logistics, 22(2), 2001, pp. 1-25.

[30] Munn, K., "Introduction: What is Ontology for?", in Applied Ontology: An Introduction, K. Munn and B. Smith, Editors. 2008. ontos: Heusenstamm, Germany.
[31] Nimmagadda, S.L., T. Reiners, and L.C. Wood, "On Modelling Big Data Guided Supply Chains in KnowledgeBase Geographic Information Systems", Procedia Computer Science, 159, 2019, pp. 1155-1164.

[32] Otto, B., J. Cirullies, and C. Schwede, "Innovation durch Kooperation in Supply Chains: Geschäftsökosysteme und die Rolle der Daten", in Digital trifft Reales, T. Wimmer and C. Grotemeier, Editors, 35. Deutscher Logistik-Kongress, Berlin, 17.-19. Oktober 2018. 2018. DVV Media: Hamburg.

[33] Otto, B., S. Steinbuß, A. Teuscher, and S. Lohmann, IDS Reference Architecture Model, International Data Spaces Association, April 2019.

[34] Popplewell, K., J. Harding, R. Poler, and R. Chalmeta, eds., Enterprise Interoperability IV, Springer London, London, 2010.

[35] Schuh, G., R. Anderl, R. Dumitrescu, A. Krüger, and M. ten Hompel, Industry 4.0 Maturity Index: Managing the Digital Transformation of Companies. Update 2020, 2020.

[36] Steinmetz, C., A. Rettberg, F.C. Ribeiro, G. Schroeder, and C.E. Pereira, "Internet of Things Ontology for Digital Twin in Cyber Physical Systems", in VIII Brazilian Symposium on Computing Systems Engineering, SBESC 2018, Salvador, Brazil, November 5-8, 2018. 2018. IEEE.

[37] Swanborn, P.G., Case study research: What, why and how?, Sage Publications, Inc, Los Angeles, California, USA, 2010.

[38] Tao, F., J. Cheng, Q. Qi, M. Zhang, H. Zhang, and F. Sui, "Digital twin-driven product design, manufacturing and service with big data", The International Journal of Advanced Manufacturing Technology, 94(9-12), 2018, pp. 3563-3576.

[39] van der Valk, H., H. Haße, F. Möller, M. Arbter, J.-L. Henning, and B. Otto, "A Taxonomy of Digital Twins", in Proceedings of the AMCIS 2020, Americas Conference on Information Systems, Editor, 26th Americas Conference on Information Systems (AMCIS), Salt Lake City, U.S.A., 10.-14.08.2020. 2020.

[40] Wagg, D., K. Worden, R. Barthorpe, and P. Gardner, "Digital Twins: State-of-The-Art Future Directions for Modelling and Simulation in Engineering Dynamics Applications", ASME J. Risk Uncertainty Part B., 2020.

[41] Werthmann, D., D. Brandwein, C. Ruthenbeck, and B. Scholz-Reiter, "Standardized information exchange in automotive distribution processes", in IFAC Proceedings Volumes, 7th IFAC Conference on Manufacturing Modelling, Management, and Control, Saint Petersburg, Russia, June 19-21, 2013.

[42] Yin, R.K., Applications of Case Study Research, 3rd edn., Sage Publications, Los Angeles, CA, USA, 2011.

[43] Zrenner, J., F.O. Möller, C. Jung, A. Eitel, and B. Otto, "Usage control architecture options for data sovereignty in business ecosystems", Journal of Enterprise Information Management, 32(3), 2019, pp. 477-495. 